\title{
PEG-BCT-100 in combination with capecitabine and oxaliplatin (PACOX) in patients with advanced hepatocellular carcinoma: A Phase I study results
}

Thomas Yau ${ }^{1 *}$, Paul N.M. Cheng ${ }^{2 *}$, Joanne Chiu ${ }^{1}$, Gerry Gin Wai Kwok ${ }^{1}$, Roland Leung ${ }^{1}$, Angela M. $\mathrm{Liu}^{2}$, Tan To Cheung ${ }^{7}$, Chi Tao $\mathrm{Ng}^{3}$

${ }^{1}$ Department of Medicine, The University of Hong Kong

${ }^{2}$ Bio-Cancer Treatment International Ltd.

${ }^{3}$ Clinical Trials Centre, The University of Hong Kong

*These authors contributed equally to this work

Address correspondence to:

Dr Thomas Yau

Department of Medicine

Queen Mary Hospital, The University of Hong Kong

102 Pokfulam Road, Hong Kong, China

Email: tyaucc@hku.hk

Telephone: (852) 22553111

Keywords: hepatocellular carcinoma, arginine, PEG-BCT-100, oxaliplatin, capecitabine

\section{Word count:}

Abstract: 286 words

Main text: words

\section{Number of tables and figures: 6}

Running title: PACOX in advanced HCC 


\section{Declarations}

\section{Funding:}

Bio-Cancer Treatment International Ltd. and HKSARG Innovation and Technology Fund (project reference UIM/257).

Conflicts of interest/Competing interests:

PC and AL are employees of Bio-Cancer Treatment International Ltd.

\section{Availability of data and material:}

The datasets analyzed during the current study are available from the corresponding author on reasonable request.

Code availability:

Not applicable

\section{Authors' contributions:}

TY and PC designed the study, performed the research, and wrote the manuscript. AL wrote the manuscript. All authors read and approved the final manuscript.

\section{Ethics approval:}

Approval was obtained from the ethics committee.

\section{Consent to participate:}

Informed consent was obtained from all individual participants included in the study.

\section{Consent to publication:}

All authors of the study gave consent to publication of this study. 


\begin{abstract}
Introduction: We investigated the safety and efficacy of PEG-BCT-100 in combination with oxaliplatin and capecitabine (PACOX) in advanced HCC patients. Methods: This was a single centre phase 1 trial to assess the safety and tolerability of PACOX. All the enrolled subjects received treatment in 3-weekly cycles: IV PEG-BCT-100 $2.7 \mathrm{mg} / \mathrm{kg}$ on day 1,8 and 15 of each cycle; oral capecitabine $1000 \mathrm{mg} / \mathrm{m}^{2}$ twice daily on day 1-14 of each cycle and IV oxaliplatin on day 1 . Three dose levels of oxaliplatin $\left(85 \mathrm{mg} / \mathrm{m}^{2}, 100 \mathrm{mg} / \mathrm{m}^{2}\right.$ or $\left.130 \mathrm{mg} / \mathrm{m}^{2}\right)$ were studied to define the maximum tolerated dose (MTD). Adverse events (AEs), efficacy by RECIST v1.1, time to progression (TTP), progression-free survival (PFS) and overall survival (OS) were studied. Results: Seventeen patients were enrolled at 3 doses of oxaliplatin: $85 \mathrm{mg} / \mathrm{m}^{2}$ (8 patients), 100 $\mathrm{mg} / \mathrm{m}^{2}$ (3 patients), and $130 \mathrm{mg} / \mathrm{m}^{2}$ (6 patients). The median age was 55 years; all had localregional chemotherapy or target therapy such as sorafenib, but no systemic chemotherapy. Most common AEs were nausea (82\%), injection site reaction (76\%), palmar-plantar erythrodysesthesia (59\%), oral mucositis (53\%) and vomiting (53\%). There was no dose-limiting toxicity (DLT). Median duration on study was 8 weeks overall. In 14 evaluable cases, one achieved partial response (PR), 4 had stable disease (SD); disease control rate was 36\% (5/14); most responses were observed in the $130 \mathrm{mg} / \mathrm{m}^{2}$ cohort with $1 \mathrm{PR}$ and 2 SDs ( $3 / 6$ or $\left.50 \%\right)$. The median TTP, PFS were both 7.0 weeks. Overall median OS was 10.7 months; the median OS was not reached at 19.4 months of follow-up in the third cohort. Conclusion: The PACOX regimen demonstrated good anti-cancer activity and survival advantage in advanced pre-treated HCC with favourable safety profile. It warrants further phase II/III studies.
\end{abstract}




\section{Introduction}

Before the use of sorafenib, the mainstay of treatment for hepatocellular carcinoma (HCC) was chemotherapy. Since early 2010, oxaliplatin-based chemotherapy regimens such as FOLFOX, because of their acceptable toxicity profiles and response rates, albeit modest, became the chemotherapy regimens of choice. In one large randomized multicenter phase III study comparing FOLFOX4 against doxorubicin in chemotherapy-naïve HCC, response rate in the FLOFOX4 arm was $8.15 \%$, median progression-free survival (PFS) in the region of 2-3 months and a median overall survival (OS) of only 6.4 months (1), which was not dissimilar to historical controls.

The first approved agent for systemic treatment of advanced HCC in the first line setting was sorafenib. In the phase 3 SHARP trial, sorafenib demonstrated significantly superior median OS (10.7 months vs 7.9 months) and time to radiological progression (5.5 months vs 2.8 months) compared to placebo as first systemic treatment for HCC (2). Since this study focused mainly on Caucasian non-hepatitis B related HCC, another study was initiated in Asia to define sorafenib's activity in Asian hepatitis B-related HCC (3). In this study, there was only a marginal OS improvement of 6 weeks (6.5 vs. 4.2) with a time to progression (TTP) improvement of 2.8 vs 1.4 months in the sorafenib arm. Similarly, lenvatinib has also been approved as first-line treatment based on the results of the phase 3 REFLECT trial, which demonstrated non inferior median survival times between lenvatinib and sorafenib (13.6 months vs 12.3 months respectively) as first systemic treatment of HCC (4). Recently, in the landmark IMBrave150 trial, atezolizumab combined with bevacizumab was compared against sorafenib in the first-line setting. In the primary analysis, the atezolizumab-bevacizumab arm had superior OS (hazard ratio for death 0.58 , $\mathrm{p}<0.001$ ), PFS (hazard ratio for disease progression or death $0.59, \mathrm{p}<0.001$ ), and overall responsr rate (ORR) (27.3\% vs $11.9 \%$ by RECIST 1.1, p<0.001) compared to the sorafenib arm. An updated analysis showed a median OS of 19.2 months of the atezolizumab-bevacizumab arm vs 13.4 months of the sorafenib arm ( $\mathrm{p}=0.0009)$. No new safety signals were identified (5). The success of IMbrave150 has several significant impacts on the treatment landscape of HCC and more importantly atezolizumab-bevacizumab has now been established as arguably the first-line treatment of choice worldwide.

A paradigm shift in the treatment of HCC emerged in the 2000s - enzyme therapy. Rationale of 
this being majority of HCC lack the arginine regeneration enzyme argininosuccinate synthetase (ASS) making them reliant on exogenous arginine for growth. Depletion of arginine in vitro and in vivo lead to rapid cell death of this type of cancer, which are termed as arginine auxotrophic cancer (6). Tumor ASS negativity could also be used as a biomarker predicting anti-tumor response (7). Currently there are two main arginine depleting agents in clinical phase development in HCC: 1) arginine deiminase (ADI-peg) is a Mycoplasma protein derivative which degrades arginine by its conversion to cirtrulline; 2) pegylated arginase (PEG-BCT-100) enzymatically depletes arginine by converting it to ornithine, then cirulline and polyamines. In a single arm phase II study, ADI-peg at two doses, 160 or $320 \mathrm{IU} / \mathrm{m}^{2}$ were randomly assigned to two groups of Asian pretreated HCC patients (8). The authors reported no objective response; disease control rates (DCRs) were similar in both groups (31\%); median OS was 7.3 months. In a global ADI-peg phase III study involving 635 cases of post-sorafenib HCC in a 2:1 randomization, ADI-peg was tested against best supportive care (BSC) (9). There was no difference in both OS (7.8 vs 7.4 months) and PFS (2.6 vs 2.6 months) between the test arm and the BSC arm. In these studies, there were no objective clinical response in test arm; however, for those who had more prolonged arginine depletion > 6 weeks, there was "an improvement in OS". It therefore appears that key to OS benefit with arginine depleting agents in HCC lies with the duration of arginine depletion.

There are three clinical studies testing the safety and efficacy of PEG-BCT-100 as monotherapy in various cancers, three in Hong Kong in HCC and one in the US in melanoma and prostate cancer. In the phase I and IIa trials in HCC conducted at the University of Hong Kong, PEG-BCT-100 was well tolerated with good pharmacokinetic/pharmacodynamic (PK/PD) profile. In these 3 clinical studies (10-12) the optimal phase II dose of PEG-BCT-100 for adequate arginine depletion (defined as undetectable circulatory arginine) was defined as $2.7 \mathrm{mg} / \mathrm{kg} /$ week across ethnicity line. The two major advantages of PEG-BCT-100 are: - 1), favorable toxicity profile and, 2), its ability to arginine deplete to undetectable levels for as long as the drug is administered. More important, in pre-clinical murine models, PEG-BCT-100 synergised with 5-florouracil (13), suggesting PEGBCT-100 in combination with other treatment options may provide better management for HCC.

In our present study (Peyglated Arginase/Capecitabine/OXaliplatin or PACOX), we aimed to define the optimal therapeutic or maximum tolerated dose (MTD) of oxaliplatin in combination 
with PEG-BCT-100.

\section{Method}

\section{Patients}

Eligible male or female adult subjects (age $\geq 18$ years) were required to have histologically or cytologically or clinically diagnosed (based on European Association for the Study of the Liver non-invasive criteria) advanced HCC not amenable to surgery or loco-regional therapy or intolerant to targeted therapy. Other eligibility criteria included an Eastern Cooperative Oncology Group (ECOG) performance status 0 or 1, Child-Pugh class A or B, adequate bone marrow, liver and renal function and normal electrocardiogram. Patients with clinically stable brain metastases for at least six months after definitive therapy were also eligible. Exclusion criteria included main portal vein tumour thrombosis, ascites uncontrolled by medication, prior treatment with systemic therapeutic agents other than targeted therapy, history of liver transplantation, history of allergic reactions to compounds of similar chemical or biological composition to capecitabine and oxaliplatin. Other exclusion criteria included dihydropyrimidine dehydrogenase deficiency, pregnancy, lactation, history of seizure disorder, infection with human immunodeficiency virus or severe concurrent infections or illness.

The study was reviewed and approved by the Institutional Review Board of the University of Hong Kong/Hospital Authority Hong Kong West Cluster, and all participants provided written informed consent. The study was conducted in accordance with the Declaration of Helsinki and International Conference on Harmonisation's Guideline for Good Clinical Practice. The ClinicalTrials.gov identifier was NCT02089633.

\section{Study Design}

The co-primary objectives of the study were to determine the MTD of oxaliplatin that in combination with PEG-BCT-100 and capecitabine and also to evaluate the TTP. Secondary objectives included AE profile associated with the regimen, to explore the DCR, which included complete response (CR), partial response (PR) and stable disease (SD), and to assess PFS and OS. 
Patient were assigned to weekly intravenous (IV) PEG-BCT-100 $2.7 \mathrm{mg} / \mathrm{kg}$, oral capecitabine $1000 \mathrm{mg} / \mathrm{m}^{2}$ twice per day for 14 days, and escalating doses of intravenous oxaliplatin on day one in a 21-day cycle duration. The initial dose of oxaliplatin was $85 \mathrm{mg} / \mathrm{m}^{2}$, and subsequent doses were $100 \mathrm{mg} / \mathrm{m}^{2}$ and $130 \mathrm{mg} / \mathrm{m}^{2}$. The oxaliplatin dose was escalated using a modified $3+3 \mathrm{design}$ with cohorts of 3 to 6 patients in each dose level - dose escalation followed the standard $3+3$ design until the highest dose level (i.e., $130 \mathrm{mg} / \mathrm{m}^{2}$ ); if the first three patients enrolled at the highest dose level did not develop a dose-limiting toxicity (DLT), an additional three patients would be enrolled at the same dose level. The MTD was defined as the highest dose level where fewer than one in six patients experienced a DLT. The DLT monitoring period was initially three full treatment cycles, but was shortened to one cycle after rapid treatment termination in seven out of eight patients accrued in the first cohort.

Patients underwent regular physical examination, ECOG performance status assessment, haematology and blood chemistry, and AEs evaluation throughout the treatment period. Patients continued the same dose level of PACOX regimen until disease progression, intolerable toxicity, death or consent withdrawal.

\section{Definition of Dose-limiting Toxicity (DLT)}

DLT was defined as any of the following treatment-related events occurring during the first 21day treatment cycle which were unable to resolve to grade 1 within two days of symptomatic treatment: (1) grade $\geq 3$ diarrhoea or any gastrointestinal toxicities, (2) grade 3 hand-foot syndrome, (3) grade 3 sensory neuropathy, (4) any clinical relevant grade $\geq 3$ toxicity, (5) prolonged granulocytopenia (grade 4 for greater than 5 days), and (6) granulocytopenia with complications (such as grade $\geq 3$ fever and mucositis).

\section{Disease Evaluation}

Tumour response was evaluated by computer tomography every 6 weeks, or earlier if clinically indicated, until disease progression using Response Evaluation Criteria in Solid Tumours (RECIST) version 1.1. Patients who had at least 4 weekly injections of PEG-BCT100 and one post-baseline radiographic tumour assessment were considered evaluable for response. 


\section{Safety Monitoring}

Toxicity was assessed through physical examination and vital signs findings, safety laboratory tests results, and graded in accordance with National Cancer Institute (NCI) Common Terminology Criteria for Adverse Events (CTCAE) version 4.0. Patients who received at least one dose of any drug were considered evaluable for safety.

\section{Statistical Analysis}

DCR was summarized for the response-evaluable patients. DCR was defined as the proportion of patients achieving a best response of complete response, partial response or stable disease. Analysis of time-to-event outcomes was performed on the MTD-evaluable patients using KaplanMeier method. TTP was defined as the duration of time from start of treatment to time of documented progression according to RECIST 1.1. PFS was defined as the duration of time from start of treatment to time of progression or death, whichever occurred first. OS was defined as the time from starting study therapy to death, with censoring on the date last known alive as of September 20, 2016, at which time all patients had completed study therapy. Statistical analysis was performed by R version 3.2.0 (R Foundation for Statistical Computing, Vienna, Austria). 


\section{Results}

Twenty seven patients were screened; 17 were eligible and enrolled in the study between April 2014 and February 2015, of whom 17 received at least 1 cycle of study treatment (3 weekly injections). Two patients were not evaluable, one because of rapidly progressive disease and the other withdrew consent. Hence 15 patients were evaluable for DLT. The study flow diagram is presented in Figure 1. The baseline demographic of these 17 treated patients are shown in Table 1. The median age was 55 years (range, 33-77 years) and all patients were ethnic Chinese. Most of the patients had chronic hepatitis B infection $(88.2 \%)$. Liver cirrhosis was present in almost half of the patients $(47.1 \%)$, majority had well

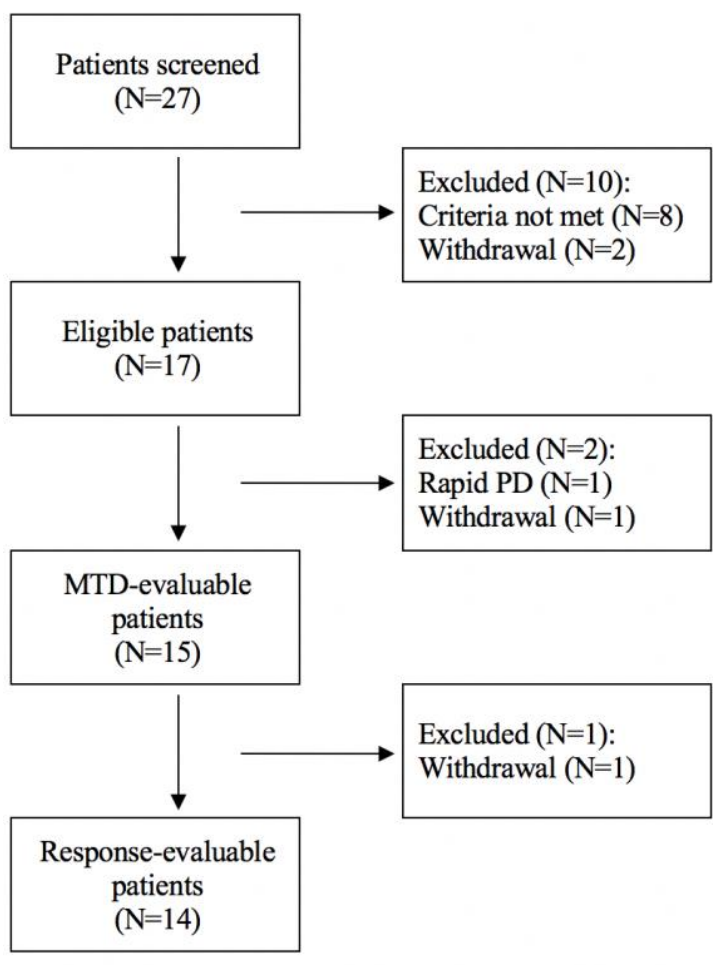

Figure 1 Study flow diagram preserved liver function (Child-Pugh class A, 94.1\%). All patients had been treated with at least one modality for HCC and prior treatment with targeted therapy was reported in $12(70.6 \%)$ patients. Of the 15 DLT-evaluable patients, one withdrew consent. Hence in total 14 patients were evaluated for treatment response. 
Table 1 Baseline demographic and disease characteristics

\begin{tabular}{|c|c|}
\hline & Overall $(\mathbf{N}=17)$ \\
\hline Age (years), median (range) & $55.4(32.9-77.2)$ \\
\hline \multicolumn{2}{|l|}{ Sex, n (\%) } \\
\hline Male & $11(64.7 \%)$ \\
\hline Female & $6(35.3 \%)$ \\
\hline \multicolumn{2}{|l|}{ Hepatitis B, n (\%) } \\
\hline Present & $15(88.2 \%)$ \\
\hline Absent & $2(11.8 \%)$ \\
\hline \multicolumn{2}{|c|}{ ECOG performance status, n (\%) } \\
\hline 0 & $11(64.7 \%)$ \\
\hline 1 & $6(35.3 \%)$ \\
\hline \multicolumn{2}{|l|}{ Liver cirrhosis, n (\%) } \\
\hline Present & $8(47.1 \%)$ \\
\hline Absent & $9(52.9 \%)$ \\
\hline \multicolumn{2}{|l|}{ Child-Pugh class, $\mathrm{n}(\%)$} \\
\hline A & $16(94.1 \%)$ \\
\hline B & $1(5.9 \%)$ \\
\hline \multicolumn{2}{|l|}{ BCLC stage, n (\%) } \\
\hline B & $3(17.6 \%)$ \\
\hline $\mathrm{C}$ & $14(82.4 \%)$ \\
\hline \multicolumn{2}{|c|}{ Prior treatment for $\mathrm{HCC}, \mathrm{n}(\%)$} \\
\hline Surgery & $13(76.5 \%)$ \\
\hline Other loco-regional therapy & $14(82.4 \%)$ \\
\hline Targeted therapy & $12(70.6 \%)$ \\
\hline - Sorafenib & $11(64.7 \%)$ \\
\hline - Other targeted therapy & $3(17.6 \%)$ \\
\hline
\end{tabular}

\section{Treatment administered}

The median number of full treatment cycles was 2 (range, <1-12). Eight (47.1\%) patients were treated with oxaliplatin at $85 \mathrm{mg} / \mathrm{m}^{2}, 3(17.6 \%)$ patients at $100 \mathrm{mg} / \mathrm{m}^{2}$ and $6(35.3 \%)$ patients at $130 \mathrm{mg} / \mathrm{m}^{2}$, in combination with PEG-BCT-100 and capecitabine.

\section{DLTs and MTD}

No patients experienced DLT at any tested dose level. The MTD of oxaliplatin was therefore 
determined at $130 \mathrm{mg} / \mathrm{m}^{2}$ when combined with weekly PEG-BCT-100 $2.7 \mathrm{mg} / \mathrm{kg}$ and capecitabine $1000 \mathrm{mg} / \mathrm{m}^{2}$ twice daily.

Treatment-related AEs (TRAEs)

TRAEs were evaluable in all intention-to-treat treat population. All patients experienced at least one gastrointestinal TRAE but the majority of TRAEs were grade 1-2, with the most common being nausea $(82.4 \%)$ associated with chemotherapy. Other common TRAEs were injection site reaction (76.5\%), palmar-plantar erythrodysesthesia $\quad(58.8 \%)$, vomiting $(52.9 \%)$, oral mucositis $(52.9 \%)$, diarrhea $(41.2 \%)$, anorexia $(41.2 \%)$, and paraesthesia (41.2\%). Among these only 1 incidence of injection site reaction was attributable to PEG-BCT-100.

Grade 3/4 TRAEs were uncommon
Table 2 Treatment-related events for all dose levels

\begin{tabular}{|c|c|c|c|}
\hline & \multicolumn{3}{|c|}{ n (\%) } \\
\hline & All grades & Grade 3 & Grade 4 \\
\hline Nausea & $14(82.4 \%)$ & 0 & 0 \\
\hline Vomiting & $9(52.9 \%)$ & $1(5.9 \%)$ & 0 \\
\hline Oral mucositis & $9(52.9 \%)$ & 0 & 0 \\
\hline Diarrhea & $7(41.2 \%)$ & $3(17.6 \%)$ & 0 \\
\hline Constipation & $4(23.5 \%)$ & 0 & 0 \\
\hline Dyspepsia & $4(23.5 \%)$ & 0 & 0 \\
\hline Abdominal pain & $1(5.9 \%)$ & 0 & 0 \\
\hline Gastroesophageal reflux disease & $1(5.9 \%)$ & 0 & 0 \\
\hline Abdominal discomfort & $1(5.9 \%)$ & 0 & 0 \\
\hline Hematemesis & $1(5.9 \%)$ & 0 & 0 \\
\hline Stomach pain & $1(5.9 \%)$ & 0 & 0 \\
\hline Injection site reaction & $13(76.5 \%)$ & 0 & 0 \\
\hline Malaise & $4(23.5 \%)$ & 0 & 0 \\
\hline Thrombocytopenia & $3(17.6 \%)$ & $2(11.8 \%)$ & 0 \\
\hline Neutropenia & $1(5.9 \%)$ & 0 & 0 \\
\hline Anorexia & $7(41.2 \%)$ & 0 & 0 \\
\hline Weight loss & $2(11.8 \%)$ & 0 & 0 \\
\hline Hypokalemia & $1(5.9 \%)$ & 0 & $1(5.9 \%)$ \\
\hline Hypoalbuminemia & $1(5.9 \%)$ & 0 & 0 \\
\hline Hyponatremia & $1(5.9 \%)$ & 0 & 0 \\
\hline Dehydration & $1(5.9 \%)$ & 0 & 0 \\
\hline Back pain & $2(11.8 \%)$ & 0 & 0 \\
\hline Pain in extremity & $1(5.9 \%)$ & 0 & 0 \\
\hline Paresthesia & $7(41.2 \%)$ & 0 & 0 \\
\hline Lethargy & $1(5.9 \%)$ & 0 & 0 \\
\hline Peripheral sensory neuropathy & $1(5.9 \%)$ & 0 & 0 \\
\hline Palmar-plantar erxthrodysesthesia & $10(58.8 \%)$ & $2(11.8 \%)$ & 0 \\
\hline Skin hyperpigmentation & $4(23.5 \%)$ & 0 & 0 \\
\hline Dry skin & $2(11.8 \%)$ & 0 & 0 \\
\hline Rash & $1(5.9 \%)$ & 0 & 0 \\
\hline Purpura & $1(5.9 \%)$ & 0 & 0 \\
\hline Nail disorder & $1(5.9 \%)$ & 0 & 0 \\
\hline
\end{tabular}

and included diarrhea (17.6\%), thrombocytopenia (11.8\%), and palmar-plantar erythrodysesthesia (11.8\%). Three treatment-related serious AEs were reported - grade 3 diarrhea, grade 3 vomiting and grade 2 hypoalbuminemia, all of which were thought to be possibly related to capecitabine and resolved with study drug interruption and/or modification. There were no deaths associated with treatment. TRAEs leading to dose reductions of oxaliplatin and capecitabine occurred in 3 and 5 patients, respectively. One patient withdrew from treatment owing to intolerable gastrointestinal toxicity from capecitabine. The TRAEs for all dose levels are summarized in Table 2. 


\section{Treatment outcomes}

Fourteen patients had at least one post-baseline radiographic tumour assessment and were evaluable for tumour response. Best responses are summarized in Table 3. One (7.1\%) patient treated with oxaliplatin at $130 \mathrm{mg} / \mathrm{m}^{2}$ had partial response and four (28.6\%) patients had stable disease, yielding an overall disease control rate of $35.7 \%$.

Table 3 Best response to treatment

\begin{tabular}{lcccc}
\hline & \multicolumn{4}{c}{ n (\%) } \\
\cline { 2 - 5 } & Cohort 1 & Cohort 2 & Cohort 3 & Overall \\
\hline $\mathrm{N}$ & 5 & 3 & 6 & 14 \\
Complete response (CR) & 0 & 0 & 0 & 0 \\
Partial response (PR) & 0 & 0 & $1(16.7 \%)$ & $1(7.1 \%)$ \\
Stable disease (SD) & $1(20.0 \%)$ & $1(33.3 \%)$ & $2(33.3 \%)$ & $4(28.6 \%)$ \\
Progression disease (PD) & $4(80.0 \%)$ & $2(66.7 \%)$ & $3(50.0 \%)$ & $9(64.3 \%)$ \\
\hline
\end{tabular}

Median TTP was 48 days (95\% confidence interval, 41-96 days) (Figure 2). For the secondary endpoints, the median PFS was 48 days (95\% confidence interval, 41-96 days). Nine patients had died at the time of analysis. Median OS was 326 days (10.7 month) (95\% confidence interval unobtainable) (Figure $3 \mathrm{a}$ and $3 \mathrm{~b}$ ).

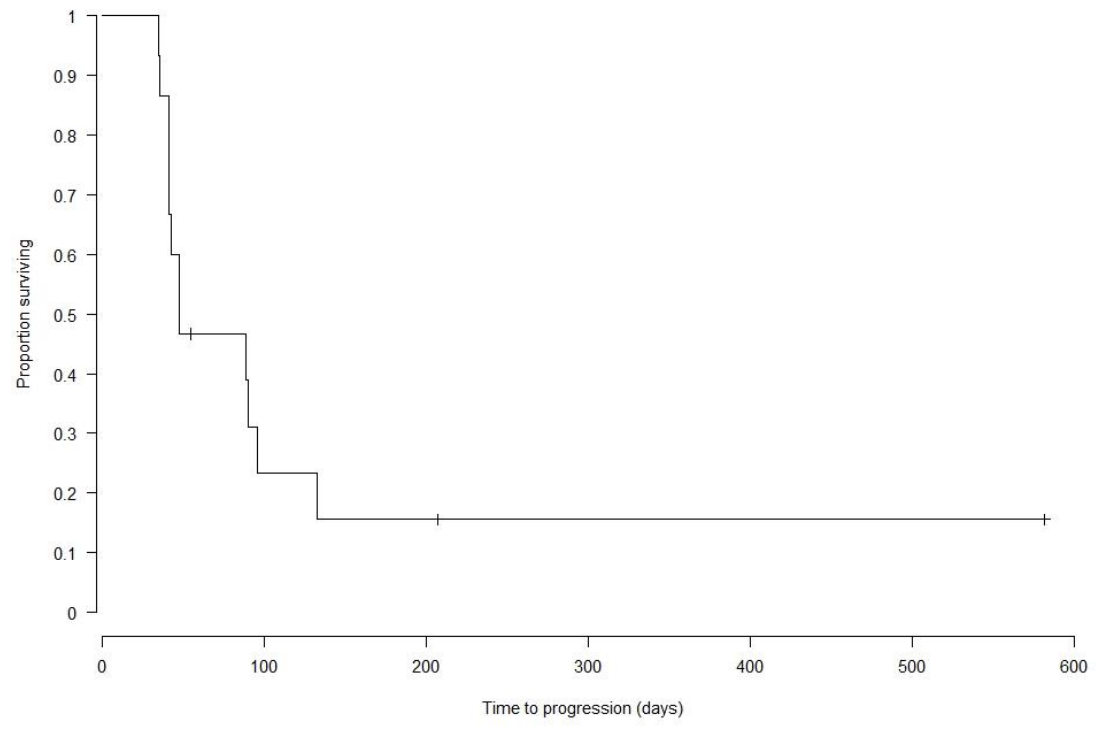

Figure 2 Time-to-progression 

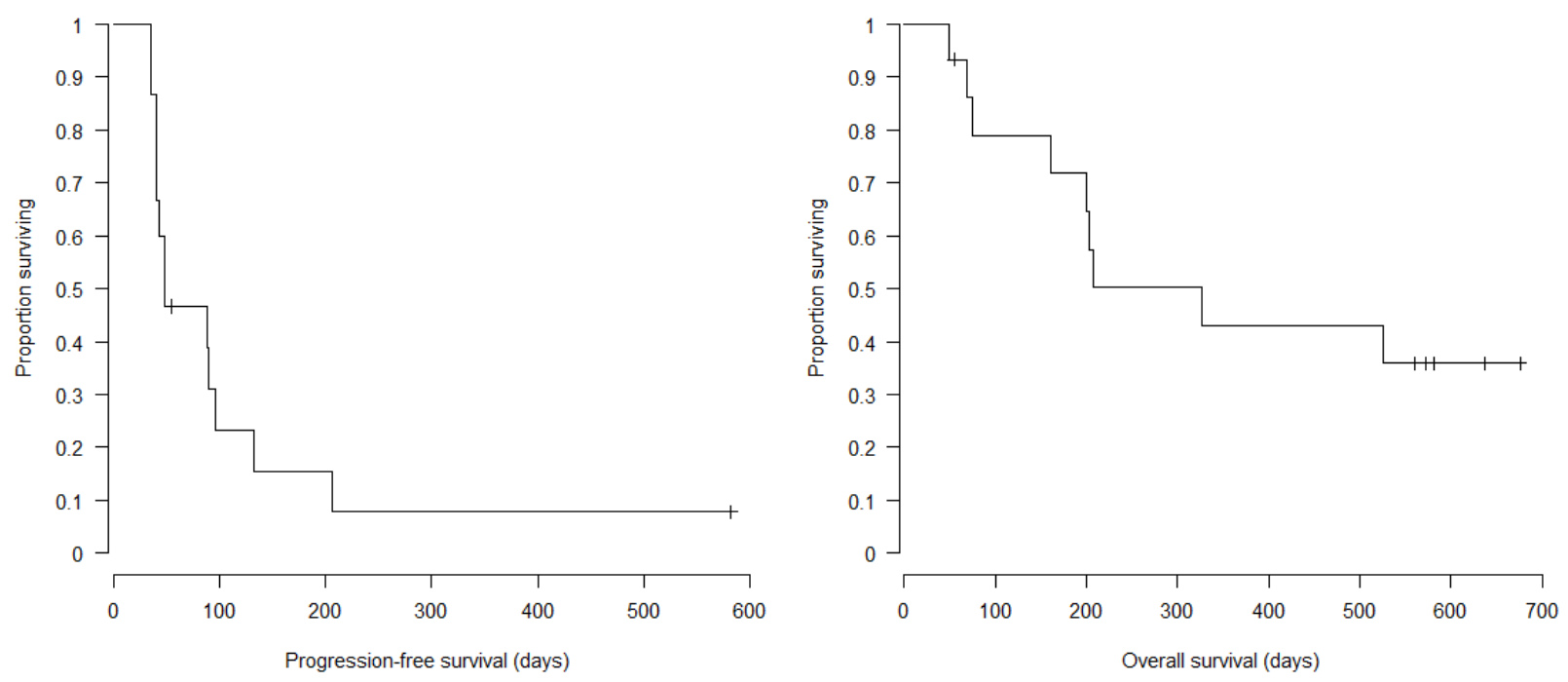

Figure 3 a) Progression-free survival and b) Overall survival

Overall survival data were analysed on all three cohorts of patients - patients were divided into two groups: 1) optimal dose oxaliplatin at $130 \mathrm{mg} / \mathrm{m}^{2}$ and 2), sub-optimal dose of oxaliplatin at $85 \mathrm{mg} / \mathrm{m}^{2}$ and $100 \mathrm{mg} / \mathrm{m}^{2}$. Kaplan-Meier analyses showed that median OS in the patient group $(\mathrm{n}=6)$ who received the optimal dose $130 \mathrm{mg} / \mathrm{m}^{2}$ was not reached after 582 days (19.4 months); in those patients $(\mathrm{n}=9)$ who received the sub-optimal doses $\left(80 \mathrm{mg} / \mathrm{m}^{2}\right.$ and $100 \mathrm{mg} / \mathrm{m}^{2}$ of oxaliplatin) had median OS of 326 days (10.7 months), although not statistically significant ( $p>$ 0.05) (Figure 4).

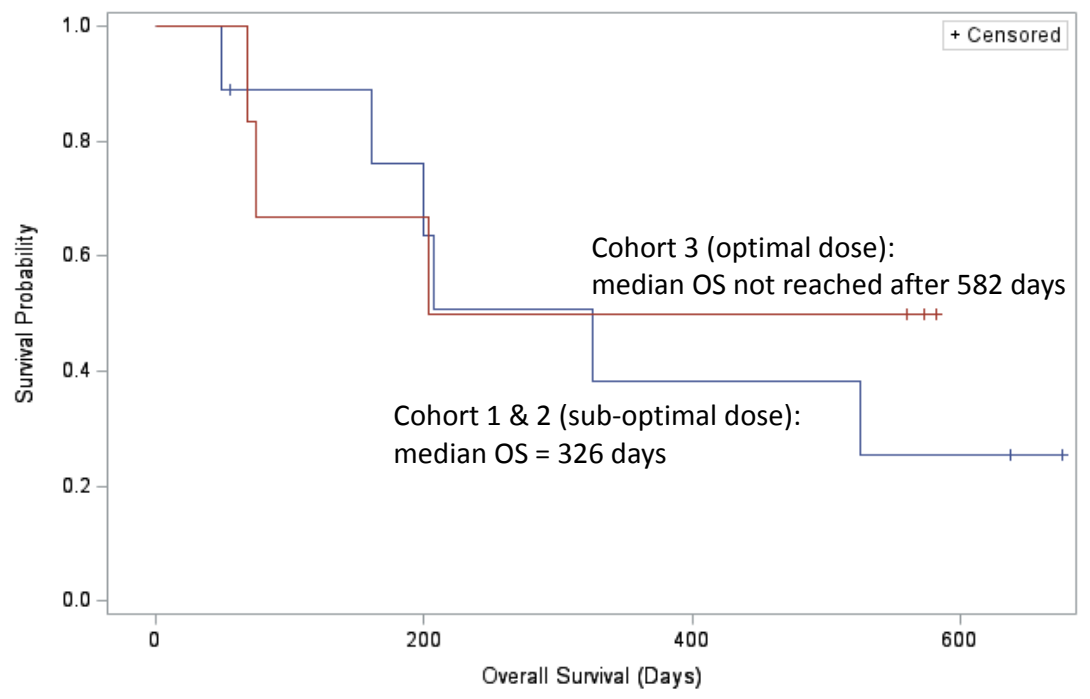

Figure 4 Post-hoc survival analysis 


\section{Discussion}

The findings in this present study might support the notion that addition of PEG-BCT-100 to oxaliplatin-based chemotherapy has additive/synergistic anti-tumor efficacy. The present dosefinding and preliminary efficacy study established $130 \mathrm{mg} / \mathrm{m}^{2}$ of oxaliplatin as the MTD for this combination regimen in advanced HCC population. Overall there were only a few adverse events over grade 2 with no DLTs (Table 2). The addition of PEG-BCT-100 in all three oxaliplatin dose levels appeared safe and tolerable. The overall DCR of 33\% with 1 PR, 4 SD and an OS of 10.7 months in all 14 evaluable cases are suggestive of clinical activities. Of note, most of the clinical responses, $1 \mathrm{PR}$ and $2 \mathrm{SD}$ were observed in the 6 subjects in the $130 \mathrm{mg} / \mathrm{m}^{2}$ dose level (Table 3) suggesting this is not only MTD dose but also maximal therapeutic dose to go forward to the next phase of clinical study. Of significance, median OS in this $130 \mathrm{mg} / \mathrm{m}^{2}$ cohort was not reached after 582 days (19.4 months) of follow-up. Implication of this is that higher dose of oxaliplatin could give higher DCR and significant OS advantage.

As for chemotherapy alone in HCC, in one large randomized phase III study comparing FOLFOX6 to doxorubicin in chemotherapy-naïve HCC, the ORR in the oxaliplatin arm $\left(130 \mathrm{~m} \mathrm{~g} / \mathrm{m}^{2}\right)$ was 8.15\% with a median OS of just 6.4 months (1). In this regard, the long OS of 19.4 months and $50 \%$ DCR in the $130 \mathrm{mg} / \mathrm{m}^{2}$ oxaliplatin dose level in the present study greatly suggest additional treatment efficacy and OS advantage from the addition of PEG-BCT-100 to oxaliplatin-based chemotherapy.

This PACOX study was one of the two clinical studies launched in 2014 to test the potential clinical efficacy of PEG-BCT-100 in combination with chemotherapy in HCC and as monotherapy to test if clinical outcomes were influenced by the tumor expression of the arginine regeneration enzyme Argininosuccinate synthetase1 (ASS1). The ASS1 study showed that OS was significantly longer in patients with HCC that under-expressed ASS1 (7). Therefore, it would be logical use ASS1 as a biomarker selection in our future PACOX study, which is now been actively planned.

As for arginine depletion therapy studies with or without chemotherapy in other ASS1- solid tumours, a literature search revealed a number of published reports: ADI-peg was tested in 
combination with various chemotherapeutic agents in a number of ASS1- cancers, including nonsmall cell lung cancer, mesothelioma and high-grade glioma $(14,15)$. In a phase I setting, ADI-peg was tested in combination with cisplatin and pemetrexed in 9 patients with ASS1- thoracic cancer which include mesothelioma and non-small cell lung cancer. Seven out of 9 patients achieved partial response which is promising (15). In a separate randomised study in which 64 ASSmesothelioma cases were randomised in a 2:1 fashion to receive single agent ADI-peg or best supportive care (14). While there was a PFS advantage in the treatment group (3.2 months vs 2.0) the OS curve crossed over showing no OS advantage. In another ASS1- high-grade glioma study, ADI-peg in conjunction with cisplatin and pemetrexed, 8 patients (80\%) had stable disease (16). Arginine depletion therefore could have additive efficacy in conjunction with chemotherapy in other ASS- tumour types.

Arginine depletion with PEG-BCT-100 leads to rapid death of auxotrophic cancers which include HCC, melanoma, acute myeloid leukaemia, chronic myeloid leukaemia, non-small cell lung cancer, mesothelioma and high-grade glioma $(6,13)$. In pre-clinical settings, anti-cancer activity of arginine depletion with PEG-BCT-100 is also augmented by chemotherapy which appears to be tumor specific, for instance, 5 FU and oxaliplatin with HCC, cytarabine with AML, pemetrexed with lung cancer and cisplatin with high-grade glioma. Hence, whether singly as monotherapy or in combination with chemotherapy, arginine depletion with PEG-BCT-100 has potential efficacies in a wide spectrum of cancers. In clinical setting, combinatorial PEG-BCT-100 with chemotherapy has gone beyond HCC - currently, there is an ongoing phase II national UK study (LI1 under BloodWise, EUDRACT no.2011-000749) testing the efficacy of combinatorial effect of PEGBCT-100 with low dose cytarabine in de novel untreated acute myeloid leukaemia over age of 65. PEG-BCT-100 is also now being tested in phase II setting as an EMA compendium study in Continental Europe and Australia in a group of pediatrics arginine auxotrophic cancers such as, neuroblastoma, sarcoma, brain tumors and acute leukemia (PARC Study, EUDRACT no. 2017002762-44). Results of these studies should be available by mid-2021.

In recent years, immunotherapy has moved into the frontline in the treatment of HCC. In March 2020, the US FDA granted the approval of dual immunotherapy nivolumab and ipilimumab for the treatment of HCC based on the demonstration of disease control rate of $33 \%$ (95\% CI, 20-48) 
in the 148 patient CheckMate-040 study (17). At a median follow up of 28 months, 4 (8\%) had a CR, and 12 (24\%) had PR with duration of response ranged from 4.6 to 30.5 months. These results are a breakthrough in the treatment of HCC (17). However, in the majority close to $70 \%$ of patients who did not response to or relapsed from this dual immunotherapy there is still an unmet medical need which novel agent such as PEG-BCT-100 in combination with chemotherapy such as PACOX could fill, particularly in developing countries where resources are limited. 


\section{References}

1. Qin S, Bai Y, Lim HY, Thongprasert S, Chao Y, Fan J, et al. Randomized, multicenter, openlabel study of oxaliplatin plus fluorouracil/leucovorin versus doxorubicin as palliative chemotherapy in patients with advanced hepatocellular carcinoma from Asia. J Clin Oncol. 2013;31:3501-8.

2. Llovet JM, Ricci S, Mazzaferro V, Hilgard P, Gane E, Blanc J-F, et al. Sorafenib in advanced hepatocellular carcinoma. N Engl J Med. 2008;359:378-90.

3. Cheng A-L, Kang Y-K, Chen Z, Tsao C-J, Qin S, Kim JS, et al. Efficacy and safety of sorafenib in patients in the Asia-Pacific region with advanced hepatocellular carcinoma: a phase III randomised, double-blind, placebo-controlled trial. Lancet Oncol. 2009;10:25-34.

4. Yamashita T, Kudo M, Ikeda K, Izumi N, Tateishi R, Ikeda M, et al. REFLECT-a phase 3 trial comparing efficacy and safety of lenvatinib to sorafenib for the treatment of unresectable hepatocellular carcinoma: an analysis of Japanese subset. J Gastroenterol. 2020;55:113-22.

5. Finn RS, Qin S, Ikeda M, Galle PR, Ducreux M, Kim T-Y, et al. IMbrave150: Updated overall survival (OS) data from a global, randomized, open-label phase III study of atezolizumab (atezo) + bevacizumab (bev) versus sorafenib (sor) in patients (pts) with unresectable hepatocellular carcinoma (HCC). JCO. Wolters Kluwer; 2021;39:267-267.

6. Scott L, Lamb J, Smith S, Wheatley DN. Single amino acid (arginine) deprivation: rapid and selective death of cultured transformed and malignant cells. Br J Cancer. 2000;83:800-10.

7. Chan SL, Cheng PNM, Liu AM, Chan LL, Li L, Chu CM, et al. A phase II clinical study on the efficacy and predictive biomarker of pegylated recombinant arginase on hepatocellular carcinoma. Invest New Drugs [Internet]. 2021 [cited 2021 Jun 8]; Available from: https://link.springer.com/10.1007/s10637-021-01111-8

8. Yang T-S, Lu S-N, Chao Y, Sheen I-S, Lin C-C, Wang T-E, et al. A randomised phase II study of pegylated arginine deiminase (ADI-PEG 20) in Asian advanced hepatocellular carcinoma patients. Br J Cancer. 2010;103:954-60.

9. Abou-Alfa GK, Qin S, Ryoo B-Y, Lu S-N, Yen C-J, Feng Y-H, et al. Phase III randomized study of second line ADI-PEG 20 plus best supportive care versus placebo plus best supportive care in patients with advanced hepatocellular carcinoma. Ann Oncol. 2018;29:1402-8.

10. Yau T, Cheng PN, Chan P, Chan W, Chen L, Yuen J, et al. A phase 1 dose-escalating study of pegylated recombinant human arginase 1 (Peg-rhArg1) in patients with advanced hepatocellular carcinoma. Invest New Drugs. 2013;31:99-107.

11. Yau T, Cheng PN, Chan P, Chen L, Yuen J, Pang R, et al. Preliminary efficacy, safety, pharmacokinetics, pharmacodynamics and quality of life study of pegylated recombinant human arginase 1 in patients with advanced hepatocellular carcinoma. Invest New Drugs. 
2015;33:496-504.

12. De Santo C, Cheng P, Beggs A, Egan S, Bessudo A, Mussai F. Metabolic therapy with PEGarginase induces a sustained complete remission in immunotherapy-resistant melanoma. $\mathrm{J}$ Hematol Oncol. 2018;11:68.

13. Cheng PN-M, Lam T-L, Lam W-M, Tsui S-M, Cheng AW-M, Lo W-H, et al. Pegylated recombinant human arginase (rhArg-peg5,000mw) inhibits the in vitro and in vivo proliferation of human hepatocellular carcinoma through arginine depletion. Cancer Res. 2007;67:309-17.

14. Szlosarek PW, Steele JP, Nolan L, Gilligan D, Taylor P, Spicer J, et al. Arginine Deprivation With Pegylated Arginine Deiminase in Patients With Argininosuccinate Synthetase 1Deficient Malignant Pleural Mesothelioma: A Randomized Clinical Trial. JAMA Oncol. 2017;3:58-66.

15. Beddowes E, Spicer J, Chan PY, Khadeir R, Corbacho JG, Repana D, et al. Phase 1 DoseEscalation Study of Pegylated Arginine Deiminase, Cisplatin, and Pemetrexed in Patients With Argininosuccinate Synthetase 1-Deficient Thoracic Cancers. J Clin Oncol. 2017;35:1778-85.

16. Hall PE, Lewis R, Syed N, Shaffer R, Evanson J, Ellis S, et al. A Phase I Study of Pegylated Arginine Deiminase (Pegargiminase), Cisplatin, and Pemetrexed in Argininosuccinate Synthetase 1-Deficient Recurrent High-grade Glioma. Clin Cancer Res. 2019;25:2708-16.

17. Yau T, Kang Y-K, Kim T-Y, El-Khoueiry AB, Santoro A, Sangro B, et al. Efficacy and Safety of Nivolumab Plus Ipilimumab in Patients With Advanced Hepatocellular Carcinoma Previously Treated With Sorafenib: The CheckMate 040 Randomized Clinical Trial. JAMA Oncol. 2020; 